\title{
Fabrication of polymer-calcite composite thin films by phase transition of vaterite composite particles with octacarboxy-terminated $\mathrm{T}_{8}$-caged silsesquioxane
}

\author{
Sakina Miyauchi, Hiroaki Imoto and Kensuke Naka
}

\begin{abstract}
Vaterite composite particles were obtained using an octacarboxy-terminated $\mathrm{T}_{8}$-caged silsesquioxane (POSS-(COOH) $\left.{ }_{8}\right)$. Incubation of the vaterite composite particles in distilled water for 3 days led to a complete phase transition to calcite. Calcite thin films were obtained on a glass substrate that was pre-coated with a poly(diallyldimethylammonium chloride) (PDDA) layer or a multilayer of PDDA with poly(sodium 4-styrenesulfonate) (PSS) through repeated cycles of layer-by-layer deposition of vaterite particles, followed by a phase transition to calcite. Free-standing calcite thin films were obtained through repeated cycles of this process after PDDA and PSS were coated on the surface of the calcite thin films. Poly(allylamine hydrochloride) (PAH) and poly (acrylic acid) (PAA) were also used instead of PDDA and PSS to prepare calcite thin films on a glass substrate. After the ionic complex formation of PAH with PAA, amide cross-linking between the two polymers can proceed through a heat treatment. In contrast to the case of using PDDA and PSS, the resulting calcite thin films did not detached from the glass substrate. The peel test revealed that the adhesion strength of the film to the glass substrate was stronger than that of scotch tape.
\end{abstract}

Polymer Journal (2016) 48, 1019-1027; doi:10.1038/pj.2016.69; published online 10 August 2016

\section{INTRODUCTION}

The nacre of shells forms layered hybrid structures based on $\mathrm{CaCO}_{3}$ and biomacromolecules. ${ }^{1,2}$ These layered hybrid structures can be compared with bricks and mortar. The bricks are flat crystals of $\mathrm{CaCO}_{3}$ that are $<1.0 \mu \mathrm{m}$ thick. ${ }^{3}$ These flat crystals of $\mathrm{CaCO}_{3}$ are not single crystals but are instead composed of nanocrystals surrounded by organic materials. ${ }^{4}$ The flat crystal units, which are separated by organic material layers, have been reported to be connected by mineral bridges. ${ }^{5,6}$ The mortar consists of biomacromolecules such as hydrophilic proteins, hydrophobic proteins and chitin. ${ }^{3}$ These hybrid structures are highly ordered microstructures ranging from a nanoscale to a macroscopic scale, and the interaction between organic and inorganic phases in these biomaterials is crucial for their extraordinary mechanical strength, structure stability and morphology. ${ }^{7}$ Moreover, the organic component, which accounts for as much as $5 \%$ of the entire weight of a biomaterial, can exert a pronounced effect on the polymorphism of the biominerals. ${ }^{8}$ The formation of macromolecules also creates lattice defects in the biomineral nanocrystals that help strengthen them against fracture by absorbing stress and halting the propagation of cracks. ${ }^{8}$ Among the various morphologies of $\mathrm{CaCO}_{3}$ micro/nanostructures, synthetic $\mathrm{CaCO}_{3}$ films are expected to be practically useful as potential barrier materials due to the low permeability of inorganic materials (such as mollusk shells). ${ }^{9}$ Although many studies have been conducted on the fabrication of $\mathrm{CaCO}_{3}$ films, ${ }^{9-12}$ few examples of free-standing $\mathrm{CaCO}_{3}$ films have been reported, ${ }^{7,13-16}$ and there are few reports on their mechanical properties. ${ }^{15,16}$

It has been reported that living organisms may use meta-stable forms of $\mathrm{CaCO}_{3}$ such as amorphous $\mathrm{CaCO}_{3}$ (ACC) or vaterite as transient precursors for the production of calcite and aragonite with hierarchically complicated structures. ${ }^{11}$ Although the use of an ACC precursor has been proposed as an attractive option for the biomimetic synthesis of a wide range of crystalline structures such as $\mathrm{CaCO}_{3}$ fibers and thin films, ${ }^{12,13}$ the use of a vaterite precursor is considered advantageous for producing calcite thin films due to the greater ease of achieving surface functionalization, size-control and isolation. Recently, we have successfully obtained calcite thin films through the layer-by-layer deposition of vaterite particles stabilized with a carboxylate-terminated poly(amidoamine)-type $\mathrm{T}_{8}$-caged silsesquioxane-core dendrimer (POSS- $\left.(\mathrm{COOH})_{16}\right)$ and poly(diallyldimethylammonium chloride) (PDDA) as a cationic polymer, followed by a phase transition to calcite. ${ }^{14,15}$ The $\mathrm{T}_{8}$-caged silsesquioxane-core dendritic structure is essential for the vaterite precursor method of fabricating calcite thin film. ${ }^{17}$ Free-standing calcite thin films were obtained through repeated cycles of this process after coating PDDA and poly(sodium 4-styrenesulfonate) (PSS) on the surface of the calcite thin films. Despite having a low organic content of 3.2 or 1.0 wt.\%, the resulting films showed clear indications of elastic bending prior to fracture.

Because the hardness and toughness of natural nacres such as abalone shell are determined by hierarchical microstructures 


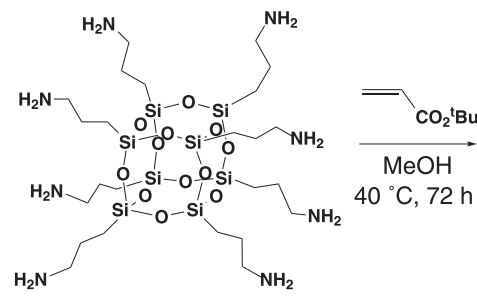

ion-exchange resin

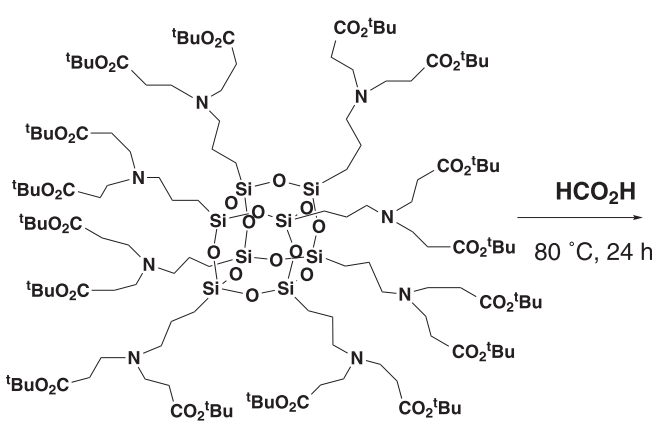

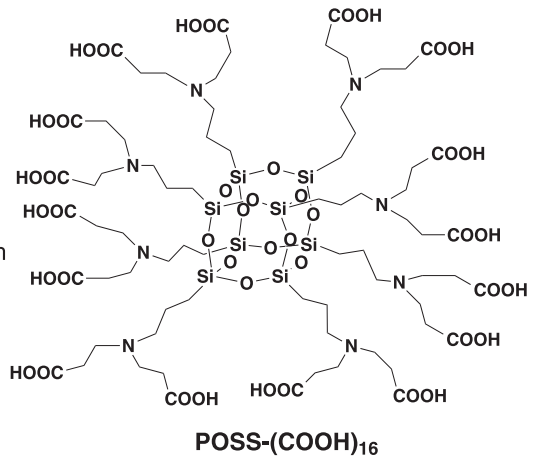

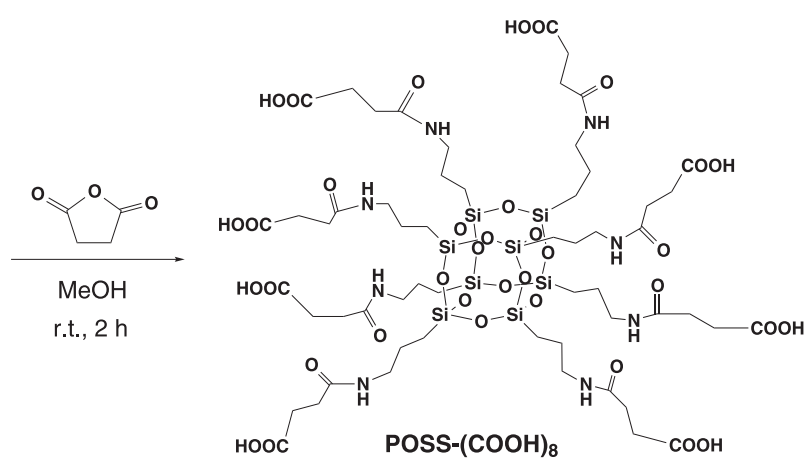

Scheme 1 Synthetic steps for carboxylate-terminated poly(amidoamine) (PAMAM)-type $\mathrm{T}_{8}$-caged silsesquioxane-core dendrimer (POSS-(COOH) 16 ) and octacarboxy-terminated $\mathrm{T}_{8}$-caged silsesquioxane (POSS-(COOH)$\left.)_{8}\right)$.

containing organic matter, it is of great importance to control the microstructures of artificial $\mathrm{CaCO}_{3}$ thin films. ${ }^{8}$ However, POSS$(\mathrm{COOH})_{16}$ requires multiple synthetic steps,${ }^{18}$ which inhibits further studies and material applications (Scheme 1). Here, we used an octacarboxy-terminated $\mathrm{T}_{8}$-caged silsesquioxane (POSS- $(\mathrm{COOH})_{8}$ ) instead of POSS- $(\mathrm{COOH})_{16}$ to prepare vaterite composite particles, because POSS- $(\mathrm{COOH})_{8}$ is obtained by a one-step reaction from octa (3-ammoniumpropyl)octasilsesquioxane octachloride. ${ }^{19} \mathrm{We}$ found that the vaterite composite particles fabricated in this study also showed a phase transition to calcite in water. Free-standing calcite thin films were obtained through repeated cycles of layer-by-layer deposition of the vaterite particles with PDDA and subsequent phase transition to calcite after PDDA and PSS layers were coated on the surface of the calcite thin films. Poly(allylamine hydrochloride) (PAH) and poly(acrylic acid) (PAA) were also applied for the vaterite precursor method instead of the PDDA and PSS layers, and we found that the multilayered calcite products did not detach from the glass substrates.

\section{EXPERIMENTAL PROCEDURE}

\section{Materials}

(3-Aminopropyl)triethoxysilane was purchased from Shin-Etsu Chemical Co., Ltd (Tokyo, Japan). Succinic anhydride was purchased from NACALAI TESQUE, Inc. (Kyoto, Japan). PSS (Mw:70 000) and PDDA (Mw:100 000200 000) were purchased from Sigma-Aldrich Japan (Tokyo, Japan). Calcium chloride and ammonium carbonate were purchased from Wako Pure Chemical Industries, Ltd (Tokyo, Japan). Glass slides (ASLAB) were purchased from AS ONE Co (Osaka, Japan). The octacarboxy-terminated $\mathrm{T}_{8}$-caged silsesquioxane (POSS- $\left.(\mathrm{COOH})_{8}\right)$ was prepared according to the reported procedure. ${ }^{19}$

\section{Instruments}

${ }^{1} \mathrm{H}$ nuclear magnetic resonance $(400 \mathrm{MHz})$ and ${ }^{29} \mathrm{Si} \mathrm{NMR}(80 \mathrm{MHz})$ spectra were recorded using a PDX-400 NMR spectrometer (Bruker Biospin, Rheinstetten, Germany). Fourier transform infrared (FT-IR) spectra were recorded using a JASCO FT/IR-4100 spectrometer (JASCO, Tokyo, Japan) by the $\mathrm{KBr}$ pellet method. The morphologies of $\mathrm{CaCO}_{3}$ particles were observed using a VE-8800 scanning electron microscope (SEM) (KEYENCE, Osaka, Japan). Thermogravimetric analysis (TGA) was measured on a TGA-50 (Shimadzu Corporation, Kyoto, Japan) to a temperature of $1000^{\circ} \mathrm{C}$ at a heating rate of $10^{\circ} \mathrm{C} \min ^{-1}$ under an air atmosphere. The zeta potential was measured using an ELSZ-1000 (Otsuka Electronics Co., Ltd., Osaka, Japan), which is based on laser-Doppler velocimetry in an electric field. The zeta potential was calculated from the electrophoretic mobility using the Smoluchowski equation. The X-ray diffraction (XRD) was recorded on a Smart Lab (Rigaku Co., Tokyo, Japan) with $\mathrm{CuK \alpha}$ radiation $(\lambda=1.5604 \AA)$ in $\theta / 2 \theta$ mode at room temperature. The $2 \theta$ scan data were collected at $0.01^{\circ}$ intervals, and the scan speed was $1^{\circ}(2 \theta / \mathrm{min})$.

\section{Precipitation of vaterite composite particles}

The standard preparation of vaterite composite particles was conducted as follows. First, a stock aqueous solution of POSS- $(\mathrm{COOH})_{8}\left(7.5 \times 10^{-2} \mathrm{mmol}\right)$ was prepared in distilled water, and the $\mathrm{pH}$ was adjusted to 11 using $1 \mathrm{M} \mathrm{NaOH}$ aqueous solution. Then, $0.50 \mathrm{ml}$ of $0.1 \mathrm{M} \mathrm{CaCl}_{2}$ aqueous solution (adjusted to $\mathrm{pH} 8.5$ with aqueous $\mathrm{NH}_{3}$ ) was added via syringe to $4.5 \mathrm{ml}$ aqueous solution of the POSS- $(\mathrm{COOH})_{8}$ under gentle stirring at $20^{\circ} \mathrm{C}$. After mixing the reaction solution for $3 \mathrm{~min}, 0.50 \mathrm{ml}$ of $0.1 \mathrm{M}\left(\mathrm{NH}_{4}\right)_{2} \mathrm{CO}_{3}$ aqueous solution (adjusted to $\mathrm{pH} 10$ with aqueous $\mathrm{NH}_{3}$ ) was added via syringe to the reaction solution. This solution was kept at $20^{\circ} \mathrm{C}$ for $1 \mathrm{~h}$ of incubation with stirring. The precipitated $\mathrm{CaCO}_{3}$ particles were collected by using a $0.1 \mu \mathrm{m}$ pore-sized membrane filter, washed several times with distilled water, and then dried at room temperature under reduced pressure.

\section{Fabricating calcite thin films using PDDA or (PDDA/PSS) multilayer}

A typical fabrication of calcite thin films was performed as follows (Figure 1). Prior to film assembly, a glass slide was cleaned by ultrasonication in acetone for $30 \mathrm{~min}$ followed by rinsing with distilled water, ultrasonication in a wash solution ( $1 \%$ potassium hydroxide, $39 \%$ distilled water and $60 \%$ ethanol) for another $30 \mathrm{~min}$ followed by rinsing with distilled water, and drying with a gentle air steam. PDDA was pre-coated three times on both sides of the cleaned 
a

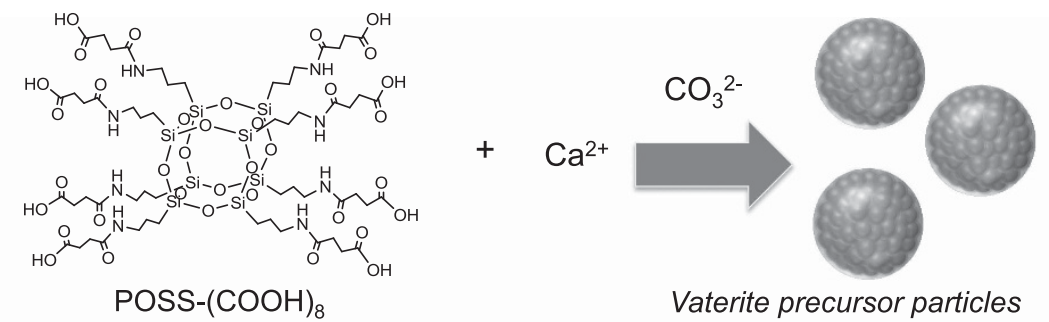

b
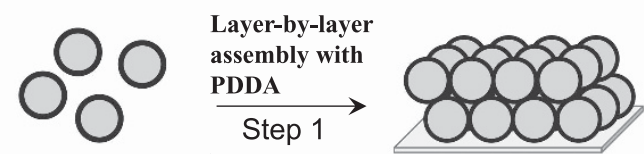

$\underset{\text { Step } 2}{\stackrel{\text { Phase transition }}{\longrightarrow}}$

Vaterite precursor particles

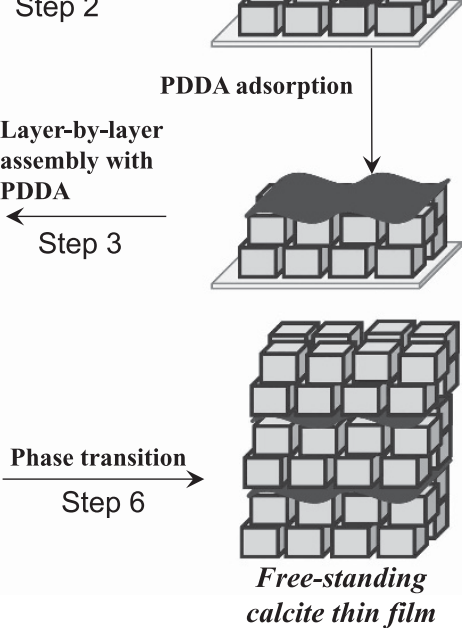

Figure 1 Schematic illustrations of (a) preparation of vaterite precursor particles with POSS- $(\mathrm{COOH})_{8}$ and (b) the layer-by-layer assembly process to produce free-standing calcite thin films.

glass substrate by immersing for $15 \mathrm{~min}$ in aqueous PDDA $\left(5.0 \mathrm{mg} \mathrm{ml}^{-1}\right)$ at room temperature, followed by rinsing with distilled water in three separated vials $(1 \mathrm{~min}$ each), and drying with a gentle air stream. The pre-coated glass substrate was alternately immersed for $15 \mathrm{~min}$ in an ethanol dispersion of vaterite composite particles $\left(4.0 \mathrm{mg} \mathrm{ml}^{-1}, \mathrm{pH} \mathrm{11}\right)$ and in an ethanol solution of PDDA $\left(1.0 \mathrm{mg} \mathrm{ml}^{-1}\right)$ at room temperature, then rinsed three times with ethanol ( $1 \mathrm{~min}$ each), followed by drying with a gentle air stream. Multilayer films were then formed by repeating this sequential adsorption process 10 times. The top surfaces of the multilayer films were coated with PDDA. The final deposition is denoted as $\mathrm{PDDA} /($ vaterite/PDDA) 10 . Calcite thin films denoted as $\mathrm{PDDA} /(\text { calcite film-A })_{1}$ were obtained by incubating the $\mathrm{PDDA} /(\text { vaterite/PDDA })_{10}$ in distilled water for 5 days at $30^{\circ} \mathrm{C}$. Then, the PDDA layer was coated on the top surface of the $\mathrm{PDDA} /($ calcite film-A) 1 by immersion in an ethanol solution of PDDA $\left(3.0 \mathrm{mg} \mathrm{ml}^{-1}\right)$ for $15 \mathrm{~min}$ at room temperature three times. The resulting layer was rinsed three times with ethanol (1 min each), followed by drying with a gentle air stream. The PDDA/(calcite film-A $)_{1}$ coated with the PDDA layer was alternately immersed for $15 \mathrm{~min}$ in an ethanol dispersion of vaterite composite particles $\left(4.0 \mathrm{mg} \mathrm{ml}^{-1}, \mathrm{pH} 11\right)$ and in an ethanol solution of PDDA $\left(1.0 \mathrm{mg} \mathrm{ml}^{-1}\right)$ at room temperature, then rinsed three times with ethanol (1 min each), followed by drying with a gentle air stream. Multilayer films were formed on the surface of PDDA/(calcite film-A $)_{1}$ by repeating this sequential adsorption process 10 times. The top surfaces of the multilayer films were coated with PDDA. The final deposition was denoted as $\mathrm{PDDA} /(\text { calcite film-A })_{1} / \mathrm{PDDA} /(\text { vaterite/PDDA })_{10}$. Subsequently, the multilayer film was incubated in distilled water for 5 days at $30^{\circ} \mathrm{C}$ to form films denoted as $\mathrm{PDDA} /(\text { calcite film-A })_{2}$. Then, a PDDA layer was again applied to facilitate the adsorption of the vaterite particles. After repeating this process 10 times to form a multilayer film and incubating in distilled water at $30^{\circ} \mathrm{C}$, a free-standing calcite thin film was obtained by tapping against a hard surface.

Alternatively, a (PDDA/PSS) $)_{2} / \mathrm{PDDA}$ multilayer was also coated on the top surface of the PDDA/(calcite film-A) ${ }_{1}$ by alternately immersing in an ethanol solution of PDDA $\left(3.0 \mathrm{mg} \mathrm{ml}^{-1}\right)$ for $15 \mathrm{~min}$ and in a ethanol/water solution of PSS $\left(3.0 \mathrm{mg} \mathrm{ml}^{-1}\right)$ for $5 \mathrm{~min}$ at room temperature.

Fabricating calcite thin films using (PAH/PAA $)_{2} / \mathrm{PAH}$ multilayer $\mathrm{A}(\mathrm{PAH} / \mathrm{PAA})_{2} / \mathrm{PAH}$ multilayer was coated on the PDDA/(calcite film-A $)_{1}$ by alternately immersing in an ethanol/water solution $(80 / 20 \%$ solvent ratio) of PAH (20 mM, pH 8.5) for $15 \mathrm{~min}$ and in an ethanol solution of PAA (20 mM, $\mathrm{pH}$ 6.5) for $15 \mathrm{~min}$ at room temperature, then rinsing three times with ethanol (1 min each). No drying step was used in the dipping procedure. The $20 \mathrm{~mm}$ concentration was based on monomer unit weight. The calcite film coated with $(\mathrm{PAH} / \mathrm{PAA})_{2} / \mathrm{PAH}$ multilayer was dried with a gentle air stream, followed by heating for $5 \mathrm{~min}$ at $200{ }^{\circ} \mathrm{C}$ to promote cross-linking derived from the amidation of PAH with PAA. The calcite multilayer films were prepared through cycle of polyelectrolyte adsorption, lamination of the vaterite composite particles and PDDA and phase transition.

\section{Three-point bending tests}

Three-point bending tests were conducted on a micro autograph (MST-1, Shimadzu) with a span of $1.0 \mathrm{~mm}$. The externally applied load was along the $c$ axis direction with a loading speed of $1.0 \mathrm{~mm} \mathrm{~min}^{-1}$. At least five specimens were repeatedly tested in each set. The specimens were $30 \mu \mathrm{m}$ in thickness and $\sim 1 \mathrm{~cm}$ in length. The thickness was determined by SEM analysis. The data on the maximum forces $(\mathrm{P})$ and maximum displacements $(\mathrm{Y})$ at fracture were used to calculate the bending strength $\left(\sigma_{\max }\right)$ and bending modulus $(E)$ without compensation.

\section{Peel test}

The peel test was conducted using a sample on a glass substrate. Incisions of $1 \mathrm{~mm} \times 1 \mathrm{~mm}$ grid size in an area of $5 \mathrm{~mm} \times 9 \mathrm{~mm}$ were made using a cutter knife. Scotch tape was placed on the surface of the sample, followed by peeling 
off quickly. Then, the adhesion strength was estimated based on ratio of the number of the peeled grids to the tested area.

\section{RESULTS AND DISCUSSION}

Preparation and characterization of vaterite composite particles POSS- $(\mathrm{COOH})_{8}$ was used to study the influence of the molar ratios of COONa in POSS- $(\mathrm{COOH})_{8}$ against $\mathrm{Ca}^{2+}$ varying from 1 to 20 , on the formation of calcium carbonate particles. After an aqueous ammonium carbonate solution was added to the reaction mixture at $30^{\circ} \mathrm{C}$, the solution became turbid. The reaction mixtures were kept at $30^{\circ} \mathrm{C}$ for $1 \mathrm{~h}$, and the products were collected (Supplementary Table S1). The $\mathrm{CaCO}_{3}$ crystal phases of the resulting products were characterized by FT-IR spectroscopy (Supplementary Figures S1 and S2). For the molar ratios $[-\mathrm{COONa}] /\left[\mathrm{Ca}^{2+}\right]=12,16$ and 20 , FT-IR analysis showed two bands at 877 and $746 \mathrm{~cm}^{-1}$, indicating the formation of vaterite (Supplementary Figure S1). A broad band at $\sim 1100 \mathrm{~cm}^{-1}$ could be assigned to the Si-O vibration derived from the POSS unit. SEM observations showed that the average particle sizes of the spheres for the molar ratios $[-\mathrm{COONa}] /\left[\mathrm{Ca}^{2+}\right]=12,16$ and 20 were $1.61 \pm 0.32, \quad 1.59 \pm 0.30 \mu \mathrm{m}$ and $1.41 \pm 0.41 \mu \mathrm{m}, \quad$ respectively (Supplementary Figure S3). No obvious difference in particle size was observed. At lower concentrations of POSS- $(\mathrm{COOH})_{8}$ $\left([\mathrm{COONa}] /\left[\mathrm{Ca}^{2+}\right]=1,2\right.$ and 8$)$, all samples showed the bands at 876 and $713 \mathrm{~cm}^{-1}$, which could be assigned to calcite co-existing with vaterite (Supplementary Figure S2). A lower temperature of $20^{\circ} \mathrm{C}$ was used to form calcium carbonate particles at the molar ratios $[-\mathrm{COONa}] /\left[\mathrm{Ca}^{2+}\right]=12$ and 16 . The average particle sizes of the former and later spheres, labeled as Particle-1 and Particle-2, decreased to $1.25 \pm 0.10$ and $1.12 \pm 0.25 \mu \mathrm{m}$, respectively (Supplementary Figure S4). The average particle sizes decreased with decreasing temperature. Both samples showed the bands at 877 and $745 \mathrm{~cm}^{-1}$, indicating vaterite formation (Supplementary Figure S5).

The sphere compositions of Particle- 1 and Particle- 2 were estimated by TGA (Supplementary Figure S6). The samples were dried under reduced pressure at room temperature for $>1$ day to remove water physically adsorbed on the particle surfaces. The weight losses at 200-600 ${ }^{\circ} \mathrm{C}$ were due to decomposition of the organic component of POSS- $(\mathrm{COOH})_{8}$. The absorbed amounts of POSS $-(\mathrm{COOH})_{8}$ in Particle- 1 and Particle-2 calculated from these data were $17.2 \mathrm{wt} \%$ and $15.2 \mathrm{wt} \%$, respectively. The observed weight loss in the temperature range between 600 and $760{ }^{\circ} \mathrm{C}$ was $35 \mathrm{wt} \%$. Based on these data, we calculated that the relative weight loss of the inorganic component, excluding the weight of the decomposed products of POSS-COOH above $600{ }^{\circ} \mathrm{C}$ was $43 \mathrm{wt} \%$. This value is in reasonable agreement with the theoretical loss of $44 \mathrm{wt} \%$ attributed to the decomposition of pure $\mathrm{CaCO}_{3}$ to $\mathrm{CaO}$. Thus, the residual amount observed at $600{ }^{\circ} \mathrm{C}$ was estimated to represent the $\mathrm{CaCO}_{3}$ content in the obtained samples.

The zeta potential of a colloidal particle is mainly determined by its surface charge. Although the calcite surface is negatively charged, vaterite surfaces are positive. ${ }^{20}$ However, negative zeta potential values ranging from -21 to $-25 \mathrm{mV}$ were detected for the vaterite particles (Particle-1 and Particle-2). These zeta potential results indicate the exposure of POSS- $(\mathrm{COOH})_{8}$ on the $\mathrm{CaCO}_{3}$ particles.

It is well known that vaterite transforms into the most thermodynamically stable form calcite via a solvent-mediated process. ${ }^{21-24}$ We studied the phase transition of Particle-1 in aqueous solution for a long incubation period at $30^{\circ} \mathrm{C}$ (Figure 2). The products obtained after incubation for 1 day in distilled water showed that the bands at 877 and $713 \mathrm{~cm}^{-1}$, assignable to calcite, co-existed with vaterite (Figure 2c). Further incubation for 4 days in distilled water led to a complete phase transition to calcite, as estimated by the FT-IR and XRD analyses (Supplementary Figure S7). The SEM observation shows irregularly shaped particles (Figure $2 \mathrm{~b}$ ). No obvious difference in the phase transition behavior was observed in the FT-IR analysis of Particle-2 (Supplementary Figure S8) compared to Particle-1, and the XRD profile of Particle-2 after incubation for 4 days shows predominant peaks corresponding to calcite and minor peaks assignable to vaterite (Supplementary Figure S9). We then used Particle-1 to fabricate calcite thin film via a layer-by-layer deposition approach.

\section{Fabrication of calcite thin films using PDDA or (PDDA/PSS) multilayer}

A glass substrate pre-coated with a PDDA layer was alternately immersed in an ethanol dispersion of vaterite particles (Particle-1) $\left(4 \mathrm{mg} \mathrm{ml}^{-1}, \mathrm{pH}=10\right)$ and in an ethanol solution of PDDA at $30^{\circ} \mathrm{C}$ and was then rinsed with ethanol three times ( 1 min each). A multilayer film denoted as $\mathrm{PDDA} /($ vaterite/PDDA) 10 was formed by repeating this sequential adsorption process 10 times, and the top surface of the multilayer film was coated with PDDA (Step 1 in Figure 1b). The deposition of the PDDA/(vaterite/PDDA) ${ }_{10}$ multilayer films was confirmed by the SEM images, and the average film a

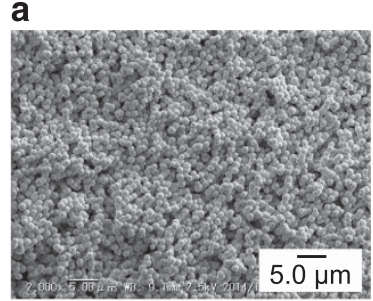

b

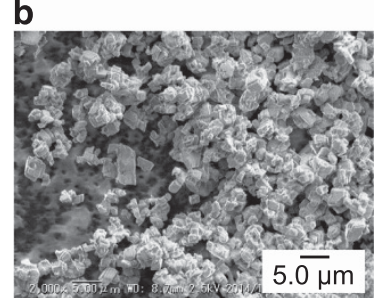

C

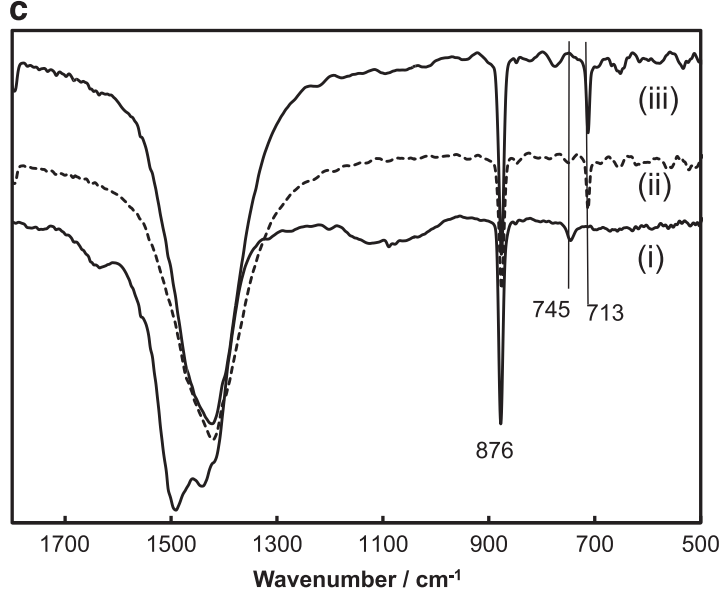

Figure $2 \mathrm{SEM}$ images for $\mathrm{CaCO}_{3}$ particles, obtained with the molar ratios $\left[-\mathrm{COONa} /\left[\mathrm{Ca}^{2+}\right]=12\right.$, (a) before and (b) after incubation in distilled water at room temperature for $4 \mathrm{~d}$; (c) FT-IR spectra for the $\mathrm{CaCO}_{3}$ particles before (i) and after incubation in distilled water at room temperature for $1 \mathrm{~d}$ (ii) and $4 \mathrm{~d}$ (iii). 


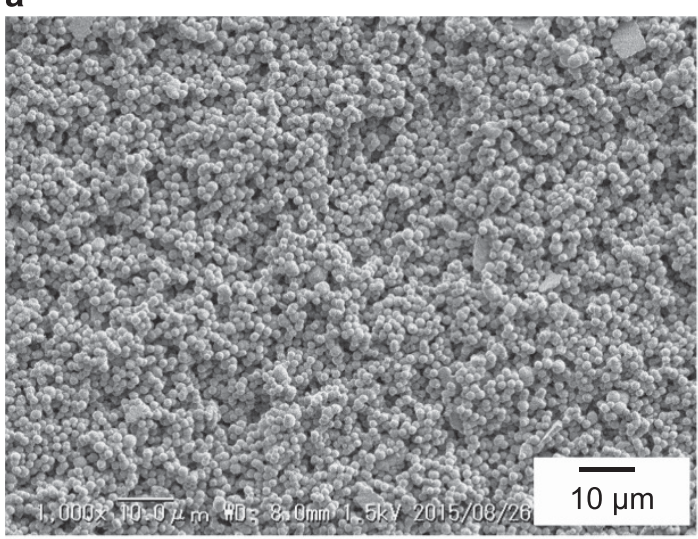

b

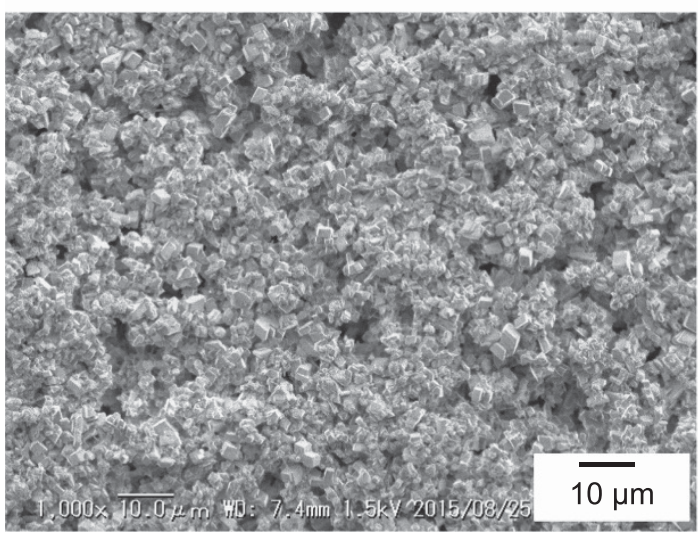

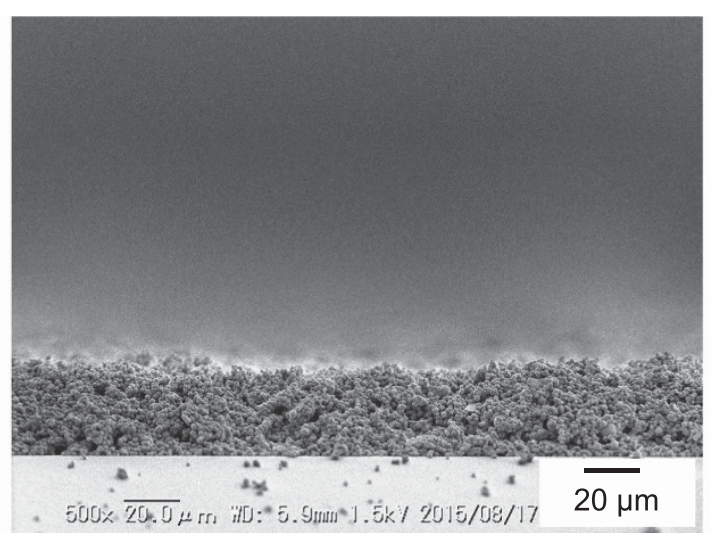

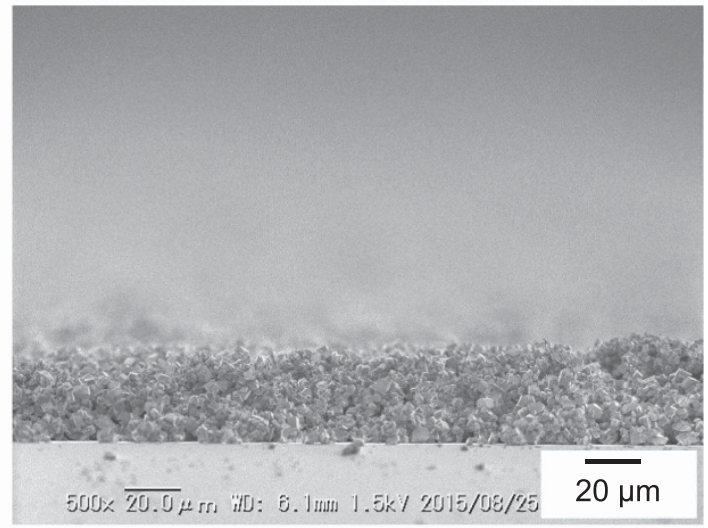

Figure 3 SEM images of surface and cross-section of PDDA/(vaterite/PDDA) 10 multilayer film before (a) and after immersion in distilled water for $5 \mathrm{~d}(\mathbf{b})$.

thickness was $30 \mu \mathrm{m}$, as estimated by the cross-section of the SEM image (Figure 3a). FT-IR analysis of the film showed two bands at 877 and $745 \mathrm{~cm}^{-1}$, which were assignable to vaterite, and a tiny band at $713 \mathrm{~cm}^{-1}$, suggesting that the multilayered film consisted of vaterite with slight amount of calcite. A broad band at $1100 \mathrm{~cm}^{-1}$ can be assigned to the Si-O vibration derived from the POSS unit (Supplementary Figure S10a). The XRD analysis of PDDA/ (vaterite/PDDA $)_{10}$ showed predominant peaks corresponding to vaterite and minor peaks assignable to calcite (Supplementary Figure S11a). The vaterite content was $94 \%$, as determined by the Reference Intensity Ratio method. ${ }^{25}$

In the FT-IR analysis, the products obtained after incubation of the $\mathrm{PDDA} /(\text { vaterite/PDDA })_{10}$ multilayer film in distilled water at $30^{\circ} \mathrm{C}$ for 4 days showed the bands at 876 and $713 \mathrm{~cm}^{-1}$, which could be assigned to calcite co-existing with vaterite. Further incubation for 5 days led to a complete phase transition to calcite (Supplementary Figure S10b). A broad band at $\sim 1100 \mathrm{~cm}^{-1}$, which could be assigned to $\mathrm{Si}-\mathrm{O}$ vibration derived from the POSS unit, was barely observed. The crystal phases of the resulting products were further confirmed by XRD analysis, suggesting that the products obtained after incubation for 5 days consisted entirely of calcite (Supplementary Figure S11b). The SEM observation of the sample after incubation for 5 days shows randomly arranged, cubic-shaped calcite particles (Figure $3 \mathrm{~b}$ ). This calcite phase is hereafter denoted as PDDA/(calcite film-A) 1 (Step 2 in Figure 1). Although some patches and pores were present in the film, the image clearly shows that a large-area continuous $\mathrm{CaCO}_{3}$ film was formed (Figure $3 \mathrm{~b}$ ). The cross-section of $\mathrm{PDDA} /(\text { calcite film-A) })_{1}$ in the SEM image showed that the average film thickness was $30 \mu \mathrm{m}$, which was the same as the $\mathrm{PDDA} /(\text { vaterite/PDDA })_{10}$ multilayer film (Figure 3a).

Deposition of multiple vaterite layers was performed on PDDA/ (calcite film-A) $)_{1}$ after a PDDA layer was applied to facilitate the adsorption of the vaterite particles, denoted as PDDA/(calcite film-A) 1 $\mathrm{PDDA} /\left(\right.$ vaterite/PDDA) ${ }_{10}$ (Step 3 in Figure 1). SEM imaging of the cross-section of the film revealed a thickness of $45 \mu \mathrm{m}$ (Supplementary Figure S12). Subsequent FT-IR analysis of this film identified two bands at 745 and $713 \mathrm{~cm}^{-1}$ corresponding to calcite and vaterite, respectively (Supplementary Figure S10c). Another incubation of the film led to a complete phase transition to calcite, as estimated by FTIR analysis (Supplementary Figure S10d), which is denoted as PDDA/ (calcite film-A) 2 (Step 4). The average film thickness of this film was $45 \mu \mathrm{m}$ (Supplementary Figure S12). Then, a PDDA layer was again applied to facilitate the adsorption of the vaterite particles. Repeating this process 10 times formed the multilayer film denoted as PDDA/ (calcite film-A) $2 / \mathrm{PDDA} /($ vaterite/PDDA) 10 (Step 5). After incubation of the resulting film in distilled water at $30{ }^{\circ} \mathrm{C}$, a free-standing calcite thin film (Film-1) was obtained by tapping it against a hard surface (Step 6) (Figure 4). The cross-section of Film-1 in the SEM images showed that the average film thickness was $\sim 54 \mu \mathrm{m}$ (Figure 5a).

The polymorphism of Film-1 was estimated by FT-IR and XRD analyses to be calcite (Supplementary Figures S10e and S13a), and its total organic component was calculated by TGA analysis to be $0.9 \mathrm{wt} \%$ (Supplementary Figure S14). A weight loss event at $200-600{ }^{\circ} \mathrm{C}$ was attributed to decomposition of the organic components of POSS- $(\mathrm{COOH})_{8}$ and PDDA. The composition of Film-1 was further probed by EDX analysis (Supplementary Figure S15 and 
a

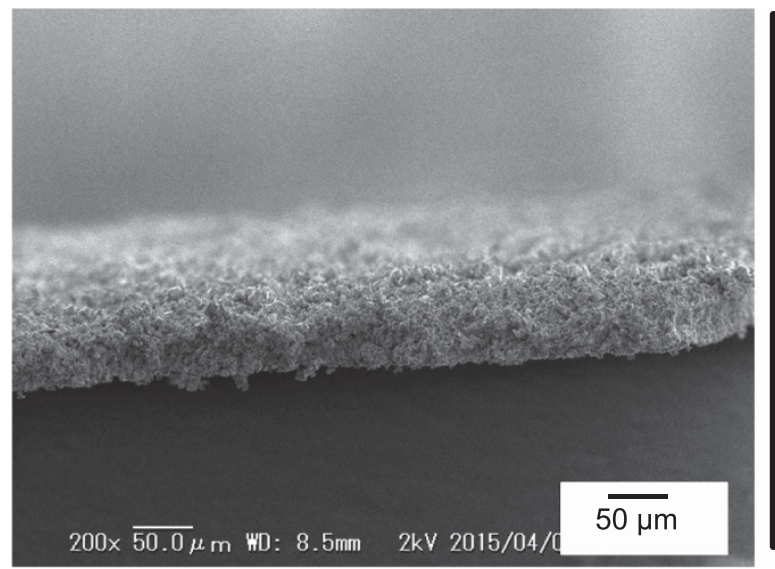

b

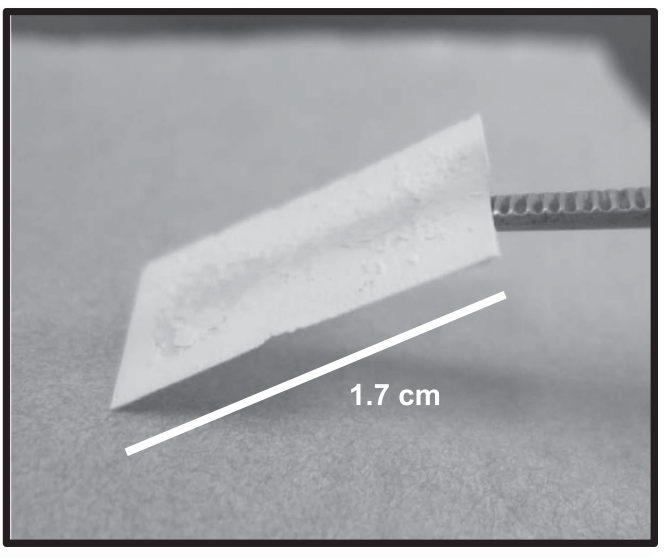

Figure 4 (a) SEM image and (b) photograph of free-standing film of Film-1.

a

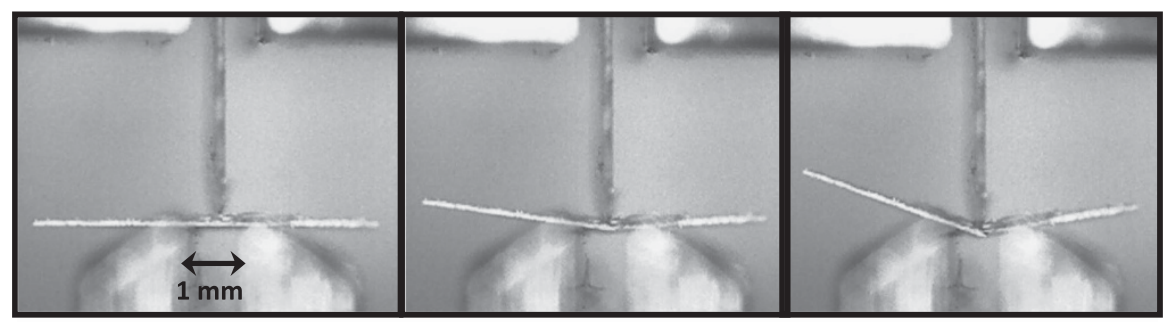

b

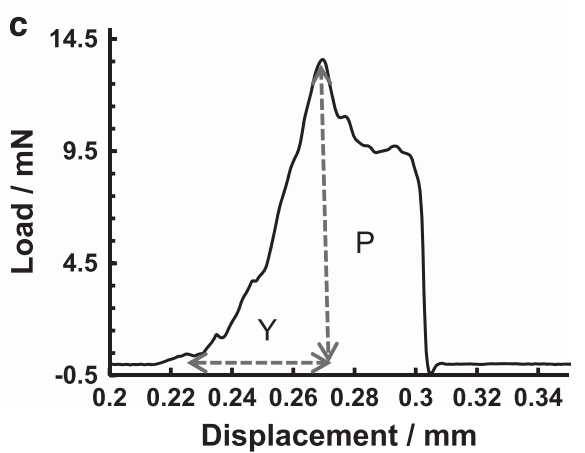

Figure 5 (a) Time series of microscopic images illustrating a three-point bending test ( $L=1 \mathrm{~mm}$ ) for Film-1. (b) Schematic illustration of a three-point bending test with the loading direction along the $c$ axis. (c) Typical load-displacement curve of Film-1. A full color version of this figure is available at Polymer Journal online.

Supplementary Table S2). Based on the distribution of elements measured by the EDX analysis at an electron accelerating voltage of $15 \mathrm{kV}, \mathrm{Ca}, \mathrm{C}$ and $\mathrm{O}$ were found to be uniformly distributed on the film. The EDX spectrum showed that a Si peak derived from POSS$(\mathrm{COOH})_{8}$ was absent in platinum-palladium-doped Film-1. These results suggested that few inorganic cage of POSS- $(\mathrm{COOH})_{8}$ were contained in the free-standing calcite thin film. In our previous our study using POSS- $(\mathrm{COOH})_{16}$, however, the EDX spectrum showed that a Si peak derived from POSS- $(\mathrm{COOH})_{16}$ was uniformly distributed on the film. ${ }^{15}$ This difference may contribute to the formation of the different calcite particle morphologies in the films.

The PDDA/(calcite film-A $)_{2}$ film was also detached from the substrate by applying stronger or more stimuli during fabrication of the PDDA/(calcite film-A $)_{2}$ film to form a free-standing calcite film (Film-2) (Supplementary Figure S16). SEM images of the cross-section of Film-2 showed that the average film thickness was $\sim 50 \mu \mathrm{m}$. A detectable weight loss of 0.2 wt $\%$ was observed between 200 and
$600{ }^{\circ} \mathrm{C}$ (Supplementary Figure S14). The polymorphism of Film-2 was estimated by the XRD analysis to be calcite (Supplementary Figure S13b).

Prior to Step 3, a (PDDA/PSS) $)_{2} / \mathrm{PDDA}$ layer was applied over the PDDA/(calcite film-A) $)_{1}$ instead of the PDDA layer to facilitate the deposition of multiple vaterite layers. A repeated incubation of the resulting multilayer film, denoted as $\mathrm{PDDA} /(\text { calcite film-A })_{1} /$ $(\mathrm{PDDA} / \mathrm{PSS})_{2} / \mathrm{PDDA} /(\text { vaterite/PDDA })_{10}$, in distilled water at $30{ }^{\circ} \mathrm{C}$ led to a complete phase transition to calcite, as estimated by FT-IR analysis (Supplementary Figure S17). The resulting calcite film was detached by breaking the glass substrate or tapping it against a hard surface to obtain a free-standing calcite thin film (Film-3) (Supplementary Figure S18). The cross-section of Film-3 in the SEM images showed that the average film thickness was $\sim 50 \mu \mathrm{m}$. The polymorphism was also confirmed by XRD analysis to be calcite (Supplementary Figure S13c). TGA analysis confirmed an organic component content of 2.2 wt $\%$ higher than in Film-1 and Film-2 (Supplementary Figure S14). 
Table 1 Organic content, thickness and results of three-point bending tests for free-standing films

\begin{tabular}{|c|c|c|c|c|c|}
\hline & Applied layer (number of calcite film-A) & Organic contenta, wt.\% & Average thickness ${ }^{b}, \mu m$ & Bending strength, $\mathrm{MPa}$ & Young's modulus, $\mathrm{MPa}$ \\
\hline Film-2 & PDDA (2) & 0.2 & 50 & $(5.7 \pm 2.5) \times 10^{-2}$ & $0.13 \pm 0.074$ \\
\hline Film-4 & PDDA-PSS (3) & 1.5 & 100 & $0.5 \pm 0.3$ & $1.3 \pm 0.4$ \\
\hline
\end{tabular}

Abbreviations: PDDA, poly(diallyldimethylammonium chloride); PSS, poly(sodium 4-styrenesulfonate). aDetermined by TGA.

bEstimated by SEM.

A glass substrate pre-coated with a (PDDA/PSS) 2 /PDDA multilayer instead of the PDDA layer used for Film-3 was employed, and the (PDDA/PSS) 2 /PDDA multilayer was used prior to Steps 3 and 5 to produce a free-standing calcite film (Film-4) (Supplementary Figure S19). In this case, the calcite film obtained after Step 4 did not detach from the glass substrate, suggesting that the pre-coated (PDDA/PSS $)_{2} / \mathrm{PDDA}$ multilayer caused a stronger interaction between the glass substrate and the calcite film. The cross-section of Film-4 in the SEM images showed that an average film thickness of $\sim 100 \mu \mathrm{m}$. The polymorphism of Film- 4 was estimated by the XRD analysis to be calcite (Supplementary Figure S13d).

Some force for the detachment of the calcite films from the glass substrates will be required for the formation of the free-standing thin films in this system. This force might be derived from the formation of network structures between the previously and the later formed calcite film. In the SEM image of the cross-section of (calcite film) ${ }_{2} /$ (vaterite) ${ }_{10} / \mathrm{PDDA}$ in the previous work, ${ }^{15}$ partial detachment from the glass substrate is observed, which supports the explanation above.

\section{Three-point bending tests}

Three-point bending tests performed on the free-standing films demonstrated that all the films cracked after several strokes when the load direction was aligned with the $c$ axis (Figure 5). By loading each sample to a maximum force $(\mathrm{P})$ at fracture, a load-displacement curve was obtained from which the maximum displacements $(Y)$ could be determined. By repeating this measurement on four specimens for each film (Supplementary Figure S20), the bending strength $\left(\sigma_{\max }\right)$ and bending modulus $(E)$ were calculated using Equations (1) and (2), respectively.

$$
\begin{gathered}
\sigma_{\max }=\frac{3 P L}{2 W h^{2}} \\
E=\frac{P L^{3}}{4 W h^{3} Y}
\end{gathered}
$$

where, $L, W$ and $h$ are the length, width, and thickness of the films. The results are summarized in Table 1 . We found that the $\sigma_{\max }$ of Film-3 is significantly higher than for Film-2, despite the difference of only $2.0 \mathrm{wt} \%$ in their respective organic material content at the same average thickness. Furthermore, the average bending modulus $(E)$ of Film-3 is also improved over Film-2. The $\sigma_{\max }$ and $E$ of Film-1 are comparable to the previously reported calcite film $(0.95 \pm 0.26 \mathrm{MPa}$ and $0.09 \pm 0.03 \mathrm{GPa}$, respectively), ${ }^{15}$ because the preparation condition was the same as for Film-1 except for using POSS- $(\mathrm{COOH})_{16}$ instead of POSS- $(\mathrm{COOH})_{8}$. Although the $\sigma_{\max }$ of Film- 1 is higher than for the previously reported calcite film, the $E$ of Film- 1 is 10 times lower. This difference may be due to the different morphology of the calcite particles in the films.

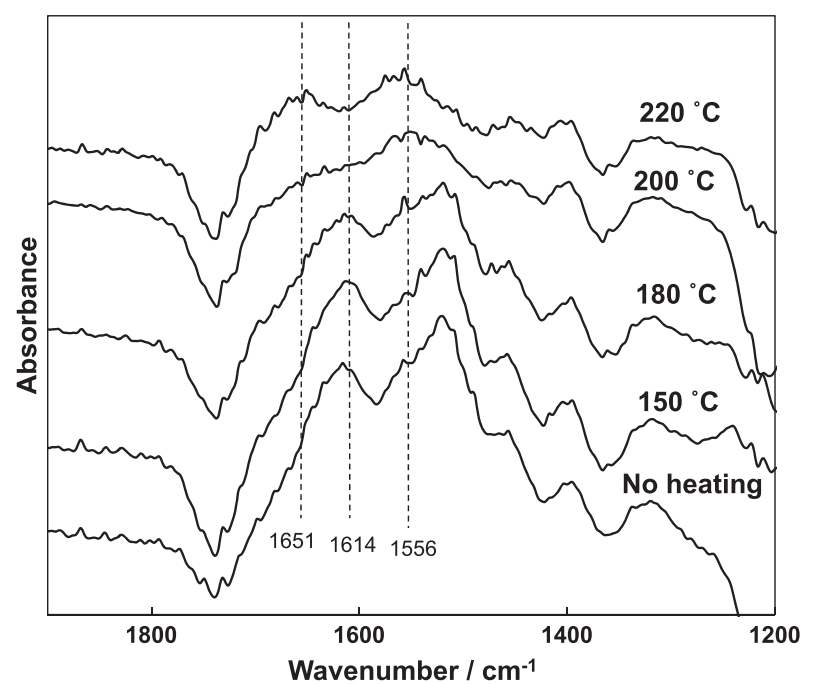

Figure 6 ATR-FT-IR spectra of (PAH/PAA) $2 / \mathrm{PAH}$ multilayers on cleaned glass substrates. Bottom line: spectrum of an untreated control sample.

Fabrication of calcite thin films using (PAH/PAA $)_{2} / \mathrm{PAH}$ multilayer PAH (Mw: 17 500) and PAA (Mw:5 000) were applied in the vaterite precursor method instead of the PDDA and PSS layers. After the ionic complex formation of PAH with PAA, amide cross-linking between the two polymers can proceed through a heat treatment. ${ }^{26-28}$ To determine the amidation temperature in this reaction condition, ATRFT-IR spectroscopy was performed on a (PAH/PAA $)_{2} / \mathrm{PAH}$ multilayer on a cleaned glass substrate, after heat treatment at temperature ranging from 150 to $220^{\circ} \mathrm{C}$ for $5 \mathrm{~min}$ (Figure 6). ATR-FT-IR analysis of the as-prepared $(\mathrm{PAH} / \mathrm{PAA})_{2} / \mathrm{PAH}$ multilayer shows an absorption band at $1612-1616 \mathrm{~cm}^{-1}$, which is assignable to asymmetric $\mathrm{NH}_{2}$. The heat-treated samples at 150 and $180^{\circ} \mathrm{C}$ still show the same absorption band. After heat treatment at 200 and $220^{\circ} \mathrm{C}$, the band at $1612-1616 \mathrm{~cm}^{-1}$ disappeared, and new bands appeared at $1651 \mathrm{~cm}^{-1}$ and $1550-1556 \mathrm{~cm}^{-1}$ corresponding to amide I and amide II, respectively, suggesting the formation of $\mathrm{CONH}^{26}$ These results suggest that charged primary amines within the multilayer react with carboxylic acids to form amides upon heating to $200^{\circ} \mathrm{C}$.

After transfer of the PDDA/(vaterite/PDDA) 10 multilayer film to the calcite film (Step 2 in Figure 1), the (PAH/PAA) $2 / \mathrm{PAH}$ multilayer was applied on the calcite film instead of the PDDA layer or the (PDDA/PSS $)_{2} / \mathrm{PDDA}$ multilayer. The calcite film coated with the $(\mathrm{PAH} / \mathrm{PAA})_{2} / \mathrm{PAH}$ multilayer was heated at $200^{\circ} \mathrm{C}$ for $5 \mathrm{~min}$ to promote cross-linking derived from amidation between $\mathrm{PAH}$ and PAA, then laminated with vaterite composite particles. Incubation of the resulting multilayer film in distilled water at $30^{\circ} \mathrm{C}$ led to a phase transition to calcite, which is denoted as (calcite film-B) $)_{2}$ (Step 4). The $(\mathrm{PAH} / \mathrm{PAA})_{2} / \mathrm{PAH}$ multilayer was then applied on the (calcite film-B $)_{2}$ film and again heated at $200^{\circ} \mathrm{C}$ for $5 \mathrm{~min}$. The lamination with 
a

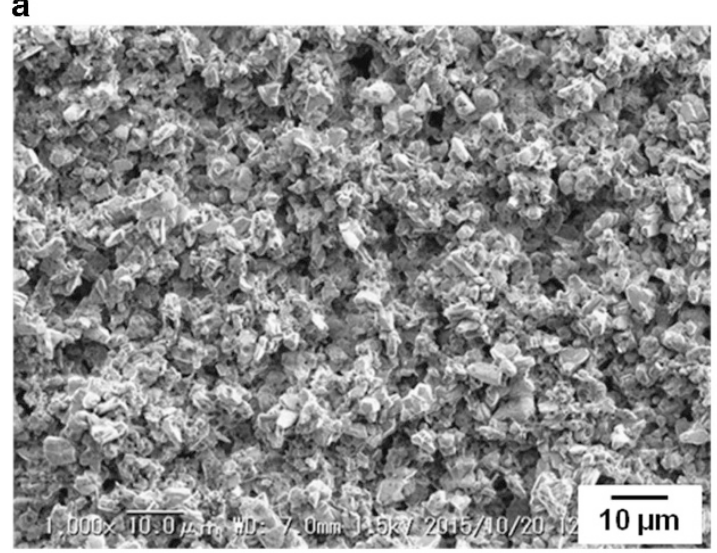

c

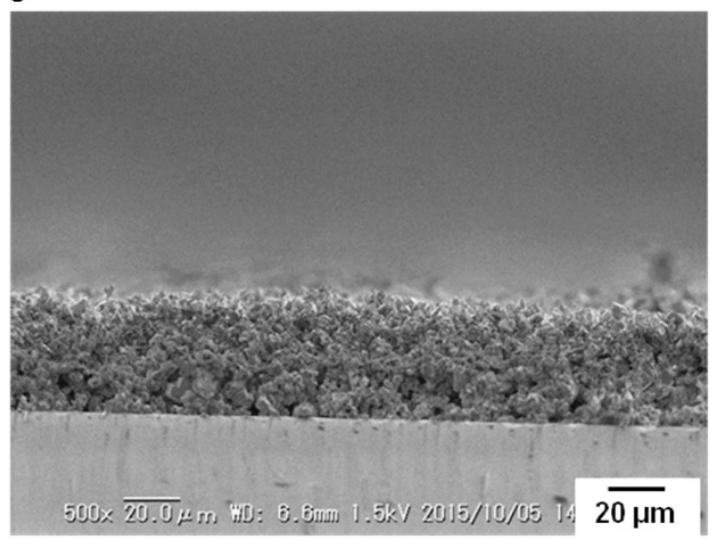

b

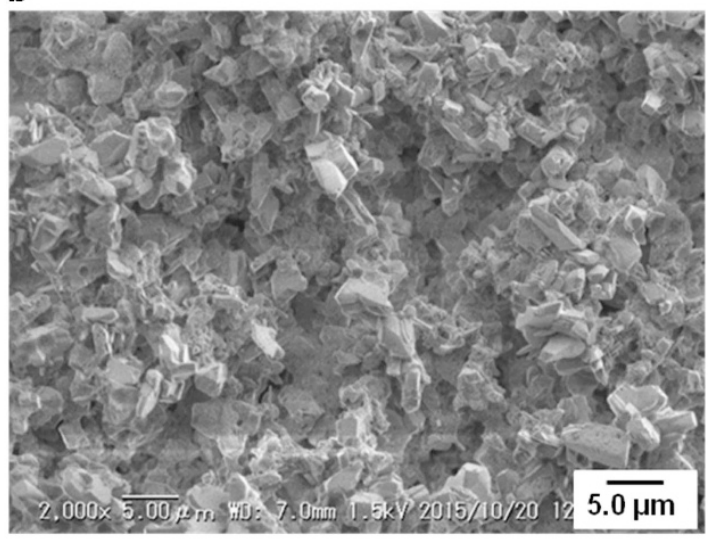

d

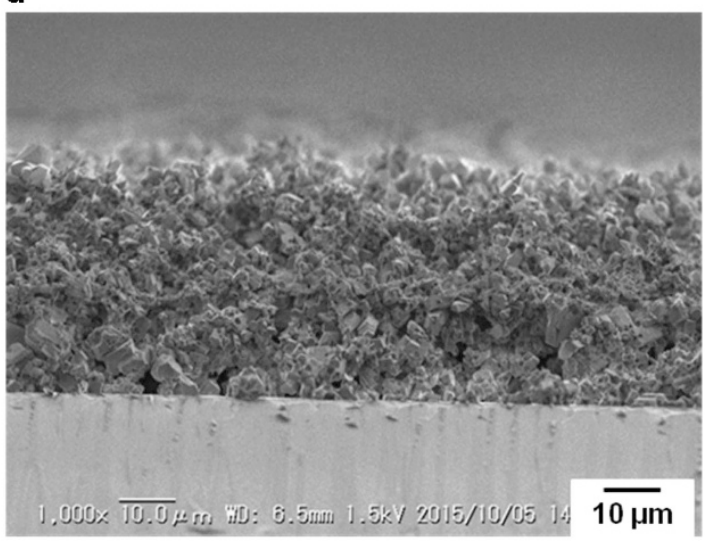

Figure 7 SEM images of (a), (b) the top surface and (c), (d) the cross-section of (calcite film-B) 3 .

a

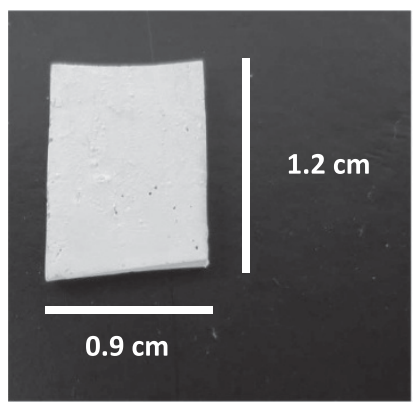

b

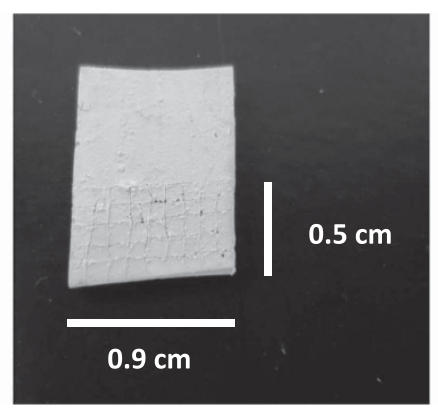

c

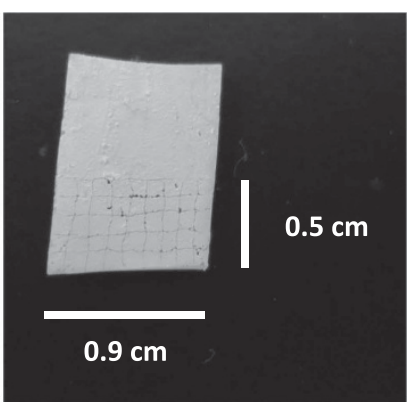

Figure 8 Photographs of (calcite film-B) $)_{3}(\mathbf{a})$ before and (b) after made incisions and (c) after peeling off scotch tape.

vaterite composite particles and the phase transition produced (calcite film-B $)_{3}$. In contrast to the case of using the (PDDA/PSS $)_{2} /$ PDDA multilayer or the PDDA layer, (calcite film-B) $)_{3}$ did not detach from the glass substrates. The formation of the calcite film was confirmed by SEM imaging (Figure 7). The SEM images of the cross-section showed an average thickness of $42 \mu \mathrm{m}$. The polymorphism was estimated by the FT-IR and XRD analysis to be calcite (Supplementary Figures S21a and S22a). TGA analysis confirmed a higher organic component content of $6.7 \mathrm{wt} \%$ than Film-3 (Supplementary Figure S23a).

The same protocol using the (PAH/PAA $)_{2} / \mathrm{PAH}$ multilayer was performed without the heat treatments at $200{ }^{\circ} \mathrm{C}$. The resulting film was brittle, and many pores were observable by the naked eye. SEM observation of this sample showed aggregates of spherical vaterite particles and no cubic crystals except on the bottom side of the film (Supplementary Figure S24). Based on SEM images of the crosssection, the average thickness of the film was $38 \mu \mathrm{m}$. FT-IR analysis of this film identified two bands at 745 and $713 \mathrm{~cm}^{-1}$, corresponding to vaterite and calcite, respectively (Supplementary Figure S22b). XRD analysis of this sample showed predominant peaks corresponding to vaterite and minor peaks assignable to calcite (Supplementary Figure S21b). TGA analysis confirmed a lower organic component content of $3.6 \mathrm{wt} \%$ compared with (calcite film-B) 3 (Supplementary Figure S23b).

The peel test was performed on the (calcite film-B) ${ }_{3}$ film. Incisions of $1 \mathrm{~mm} \times 1 \mathrm{~mm}$ grid size were made in an area of $5 \mathrm{~mm} \times 9 \mathrm{~mm}$ on the sample using an utility knife (Figure 8 ). Scotch tape was placed on the surface of the sample, and was peeled off quickly. After the peel 
test, all parts of the (calcite film-B $)_{3}$ film remained on the glass substrate (Figure 8c). Thus, the peel test revealed that the adhesion strength of the (calcite film-B) ${ }_{3}$ film to the glass substrate was greater than the adhesion strength of the scotch tape to the surface of the (calcite film) $)_{3}$ film. Applying the cross-linked (PAH/PAA) $2 / \mathrm{PAH}$ multilayer increased the amount of the organic components in the calcite film, which might inhibit the detachment of the (calcite film-B $)_{3}$ from the glass substrate.

\section{CONCLUSIONS}

Vaterite composite particles were obtained using POSS- $(\mathrm{COOH})_{8}$. Incubation of the vaterite composite particles in distilled water for 3 days led to a complete phase transition to calcite. Negative zeta potential values ranging from -21 to $-25 \mathrm{mV}$ were detected for the vaterite particles, indicating that POSS $-(\mathrm{COOH})_{8}$ was exposed on the $\mathrm{CaCO}_{3}$ particles. Free-standing calcite thin films containing a few wt $\%$ of organic polymer were obtained through repeated cycles of layer-bylayer deposition of the vaterite particles stabilized with POSS- $(\mathrm{COOH})_{8}$, followed by a phase transition to calcite, after the coating the surface of the calcite thin films with a (PDDA/PSS $)_{2} / \mathrm{PDDA}$ multilayer or PDDA layer. Despite having low organic content, the resulting films showed clear indications of elastic bending prior to fracture. PAH and PAA were also used in the vaterite precursor method in place of the PDDA and PSS layers. After the ionic complex formation of PAH with PAA, amide cross-linking between the two polymers can proceed by heat treatment. In contrast to the case using the (PDDA/PSS $)_{2} / \mathrm{PDDA}$ multilayer or the PDDA layer, the calcite multilayered products did not detach from the glass substrates. The peel test revealed that the adhesion strength of the calcite film to the glass substrate was greater than the adhesion strength of the scotch tape. The cross-linked (PAH/PAA) $2 / \mathrm{PAH}$ multilayer contributed to peeling resistance for the calcite thin films coated on the glass substrates.

\section{CONFLICT OF INTEREST}

The authors declare no conflict of interest.

\section{ACKNOWLEDGEMENTS}

This work was partially supported by a Grant-in-Aid for Scientific Research on Innovative Areas 'New Polymeric Materials Based on Element-Blocks (No.2401)' (24102003) of The Ministry of Education, Culture, Sports, Science and Technology, Japan. We thank Shimadzu Co. for performing the threepoint bending tests.

1 Estroff, L. A. \& Hamilton, A. D. At the interface of organic and inorganic chemistry: bioinspired synthesis of composite materials. Chem. Mater. 13, 3227-3235 (2001).

2 Meldrum, F. C. \& Cölfen, H. Controlling mineral morphologies and structures in biological and synthetic systems. Chem. Rev. 108, 4332-4432 (2008).

3 Imai, H., Oaki, Y. \& Kotachi, A. A Biomimetic approach for hierarchically structured inorganic crystals through self-organization. Bull. Chem. Soc. Jpn. 79 1834-1851 (2006)
4 Yao, H.-B., Fang, H.-Y., Wang, X.-H. \& Yu, S.-H. Hierarchical assembly of micro-/nanobuilding blocks: bio-inspired rigid structural functional materials. Chem. Soc. Rev. 40, 3764-3785 (2011).

5 Naka, K. \& Chujo, Y. Control of crystal nucleation and growth of calcium carbonate by synthetic substrates. Chem. Mater. 13, 3245-3259 (2001).

6 Takahashi, K., Yamamoto, H., Onoda, A., Doi, M., Inaba, T., Chiba, M., Kobayashi, A., Taguchi, T., Okamura, T. \& Ueyama, N. Highly oriented aragonite nanocrystalbiopolymer composites in an aragonite brick of the nacreous layer of Pinctada fucata. Chem. Commun. 40, 996-997 (2004).

7 Kato, T., Sugawara, A. \& Hosoda, N. Calcium carbonate organic hybrid materials. Adv. Mater. 14, 869-877 (2002).

8 Okumura, T., Suzuki, M., Nagasawa, H. \& Kogure, T. Localization of intracrystalline organic macromolecules in mollusk shell. Cryst. Growth Design 12, 224-230 (2011).

9 Kim, S., Ku, S. H., Lim, S. Y., Kim, J. H. \& Park, C. B. Graphene-biomineral hybrid materials. Adv. Mater. 23, 2009-2014 (2011).

10 Dong, L., Yang, L., Wang, Y. \& Han, M. Free-standing crystalline $\mathrm{CaCO}_{3}$ films composed of three-dimensional microstructures with different morphologies. J. Cryst. Growth 343, 86-94 (2012)

11 Cölfen, H. \& Mann, S. Higher-order organization by mesoscale self-assembly and transformation of hybrid nanostructures. Angew. Chem. Int. Ed. $\mathbf{4 2}$, 2350-2365 (2003).

12 Addadi, L., Moradiam, J., Shay, E., Maroudas, N. G. \& Weiner, S. A chemical model for the cooperation of sulfates and carboxylates in calcite crystal nucleation: Relevance to biomineralization. Proc. Natl Acad. Sci. USA 84, 2732-2736 (1987).

13 Gower, L. B. Biomimetic model systems for investigating the amorphous precursor pathway and its role in biomineralization. Chem. Rev. 108, 4551-4627 (2008).

14 Nakamura, S. \& Naka, K. Size-controlled vaterite composite particles with POSS-core dendrimer for fabrication of calcite thin films by phase transition. Langmuir 29 15888-15897 (2013).

15 Nakamura, S. \& Naka, K. Bendable, free-standing calcite thin films. Langmuir 31, 2014-2018 (2015).

16 Saito, T., Oaki, Y., Nishimura, T., Isogai, A. \& Kato, T. Bioinspired stiff and flexible composites of nanocellulose-reinforced amorphous $\mathrm{CaCO}_{3}$. Mater. Horiz. 1 , 321-325 (2014).

17 Tanaka, Y. \& Naka, K. A carbonate controlled-addition method for size-controlled calcium carbonate spheres by carboxylic acid terminated poly(amidoamine) dendrimers. Polym. J. 42, 676-683 (2010).

18 Naka, K., Fujita, M., Tanaka, K. \& Chujo, Y. Water-soluble anionic POSS-core dendrimer: synthesis and copper(II) complexes in aqueous solution. Langmuir 23 9057-9063 (2007).

19 Tanaka, K., Ishiguro, F. \& Chujo, Y. Thermodynamic study of POSS-based ionic liquids with various numbers of ion pairs. Polym. J. 43, 708-713 (2011).

20 Sawada, K. The mechanisms of crystallization and transformation of calcium carbonates. Pure Appl. Chem. 69, 921-928 (1997).

21 Ogino, T., Suzuki, T. \& Sawada, K. The rate and mechanism of polymorphic transformation of calcium carbonate in water. J. Cryst. Growth 100, 159-167 (1990).

22 Perić, J., Vučak, M., Krstulović, R., Brečević, Lj. \& Kralj, D. Phase transformation of calcium carbonate polymorphs. Thermochim. Acta 277, 175-186 (1996).

23 Jiménez-López, C., Caballero, E., Huertas, F. J. \& Romanek, C. S. Chemical, mineralogical and isotope behavior, and phase transformation during the precipitation of calcium carbonate minerals from intermediate ionic solution at $25^{\circ} \mathrm{C}$. Geochim. Cosmochim. Acta 65, 3219-3231 (2001).

24 Wolf, G. \& Günther, C. Thermophysical investigations of the polymorphous phases of calcium carbonate. J. Therm. Anal. Cal 65, 687-698 (2001).

25 Hillier, S. Accurate quantitative analysis of clay and other minerals in sandstones by XRD: Comparison of a Rietveld and a reference intensity ratio (RIR) method and the importance of sample preparation. Clay Miner. 35, 291-928 (2000).

26 Harris, J. J., DeRose, P. M. \& Bruening, M. L. Synthesis of passivating, nylon-like coatings through cross-linking of ultrathin polyelectrolyte films. J. Am. Chem. Soc. 121, 1978-1979 (1999)

27 Jang, W.-S., Jensen, A. T. \& Lutkenhaus, J. L. Confinement effects on cross-linking within electrostatic layer-by-layer assemblies containing poly(allylamine hydrochloride) and poly(acrylic acid). Macromolecules 43, 9473-9479 (2010).

28 Gustafsson, E., Hedberg, J., Larsson, P. A., Wågberg, L. \& Johnson, M. Vibrational sum frequency spectroscopy on polyelectrolyte multilayers: Effect of molecular surface structure on macroscopic wetting properties. Langmuir 31, 4435-4442 (2015).

Supplementary Information accompanies the paper on Polymer Journal website (http://www.nature.com/pj) 